\title{
Editorial: Membrane and Cytoskeleton Mechanics
}

\author{
Yuan $\operatorname{Lin}^{1 *}$ and Ying $\mathrm{Li}^{2}$ \\ ${ }^{1}$ Department of Mechanical Engineering, The University of Hong Kong, Hong Kong, China, ${ }^{2}$ Department of Mechanical \\ Engineering and Polymer Program, Institute of Materials Science, University of Connecticut, Storrs, CT, United States
}

Keywords: cell membrane, cytoskeleton, cell mechanics, modeling, experiment, cell biophysics

Editorial on the Research Topic

\section{Membrane and Cytoskeleton Mechanics}

As two main structural components of a cell, the lipid bilayer membrane [1] and cytoskeleton [2] are responsible for stabilizing the cell shape as well as playing key roles in different cellular functions. For example, important processes like cell migration [3-6], division [7, 8], and embryo development [9, 10] are all driven by internal forces generated within the cytoskeleton. In addition, various mechanosensitive proteins in the membrane allow cells to probe their microenvironment and perform functions such as mechano-transduction [11, 12] and volume regulation [13, 14]. For these reasons, extensive efforts have been devoted in the past few decades to elucidating key factors dictating the mechanical response of the lipid membrane and cytoskeleton [15-18] as well as connecting them to the capability of cells to perform biological duties or the progression of diseases such as cancer [19] and malaria [20]. This research topic intends to present a collection of substantial advances made in this field recently.

The paper by Burgos-Bravo et al. shows that proteoglycan receptor Syndecan- 4 can form a ternary complex with $\alpha_{\mathrm{V}} \beta_{3}$ integrin and the neuronal protein Thy-1, increase the lifetime of Thy-1 $-\alpha_{\mathrm{V}} \beta_{3}$ integrin binding, modulate their mechano-transduction function, and eventually result in faster neurite retraction and suppressed neurite outgrowth under pro-inflammatory conditions.

The paper by Ricketts et al. focuses on how ionic conditions influence the rearrangement of networks of actin and microtubules. Specifically, the authors show that the bulk contraction of both actin and actin-microtubule networks increases when $\mathrm{Mg}^{2+}$ concentrations varies from 2 to $20 \mathrm{mM}$. Interestingly, actin networks begin to contract at lower $\mathrm{Mg}^{2+}$ concentrations and shorter times than actin-microtubule networks. These results shed light on how varying environmental conditions can dynamically tune the morphology of the cytoskeleton without the help of motor proteins.

The paper by Bashirzadeh et al. discusses how the properties of actin crosslinkers and surrounding confinement geometry regulate the formation of actin bundles and bundle networks. Specifically, depending on the size of giant unilamellar vesicles (GUVs) encapsulating actin and concentration of fascin, a short crosslinker, straight F-actin bundles, or crosslinked bundle networks can be formed. In addition, the authors also show that the presence of a long crosslinker, $\boldsymbol{\alpha}$-actinin, impacts fascininduced GUV shape changes and significantly impairs the formation of filopodia-like protrusions.

The paper by Dasanna et al. presents how the contrast/ratio of viscosities between RBC cytosol and

Received: 01 December 2021 Accepted: 09 December 2021 Published: 04 January 2022

Citation:

Lin Y and Li Y (2022) Editorial: Membrane and

Cytoskeleton Mechanics.

Front. Phys. 9:827025.

doi: 10.3389/fphy.2021.827025 blood plasma affects the shape and dynamics of RBCs via mesoscopic hydrodynamics simulations. Interestingly, it is found that distinct RBC shapes, including tumbling cells, parachutes, and tank-treading slippers, can all appear depending on the values of viscosity contrast, flow rate, and tube diameter. Furthermore, the authors demonstrate that the tumbling-to-slipper transition, as well as the size of the parachute region, is strongly affected by viscosity contrast.

The paper by Galluzzi et al. discusses the influence of photothermal effects of fluorescent dyes on the mechanical response and stability of membranes. Specifically, the authors show a 30 min laser 
irradiation, with intensity similar to that in a typical confocal scanning microscopy experiment, is enough to trigger a $\sim 40 \%$ decrease in the breakthrough force of the stained phospholipid bilayer membrane along with a $\sim 30 \%$ reduction in its apparent elastic modulus, highlighting the importance of considering such effect when using, for example, atomic force-fluorescence correlative microscopy to investigate the deformability and permeability of cell membranes.

The paper by Schmidt and Kierfeld proposes a chemomechanical model of microtubule dynamics on the dimmer level. By taking into account the attachment/detachment and hydrolysis of tubulin dimers, the deformation of protofilaments, and the rupture/ formation of lateral bonds between them, the authors show the model is computationally efficient to capture repeated catastrophes and rescues at realistic tubulin concentrations and hydrolysis rates, as well as gain insight into microscopic structural features triggering these events.

The paper by Chen et al. discusses the organization of F-actin and viscoelasticity distribution in migrating cells in response to $\mathrm{pH}$ changes in the microenvironment. In particular, by using multifrequency atomic force microscopy (AFM) with amplitude modulation-frequency modulation (AM-FM), the authors show that, unlike the randomly distributed F-actin and the homogeneous viscoelasticity at the normal $\mathrm{pH}$ level, living Huh-7 cancer cells with the reduced $\mathrm{pH}$ level of 6.5 exhibits highly oriented and organized F-actin along the lamellipodium direction coupled with a spatial gradient both in elasticity and viscosity of the cell.

The paper by Castaneda et al. reviews recent progress on understanding how different environmental factors contribute to actin bundle assembly, organization, and mechanics. Specifically, the effects of macromolecular crowding, cation interactions, and actin-crosslinking proteins on F-actin bundling, higher-ordered

\section{REFERENCES}

1. Alberts B. Molecular Biology of the Cell. 4th ed. New York: Garland Science (2002).

2. Fletcher DA, Mullins RD. Cell Mechanics and the Cytoskeleton. Nature (2010) 463:485-92. doi:10.1038/nature08908

3. Theriot JA, Mitchison TJ. Actin Microfilament Dynamics in Locomoting Cells. Nature (1991) 352:126-31. doi:10.1038/352126a0

4. Lin Y, Shenoy VB, Hu B, Bai L. A Microscopic Formulation for the ActinDriven Motion of Listeria in Curved Paths. Biophysical J (2010) 99:1043-52. doi:10.1016/j.bpj.2010.06.001

5. Mogilner A, Oster G. Force Generation by Actin Polymerization II: The Elastic Ratchet and Tethered Filaments. Biophysical J (2003) 84:1591-605. doi:10.1016/s0006-3495(03)74969-8

6. Lin Y. Mechanics Model for Actin-Based Motility. Phys Rev E Stat Nonlin Soft Matter Phys (2009) 79:021916. doi:10.1103/PhysRevE.79.021916

7. Heng Y-W, Koh C-G. Actin Cytoskeleton Dynamics and the Cell Division Cycle. Int J Biochem Cel Biol (2010) 42:1622-33. doi:10.1016/j.biocel.2010.04.007

8. Brust-Mascher I, Civelekoglu-Scholey G, Kwon M, Mogilner A, Scholey JM. Model for Anaphase B: Role of Three Mitotic Motors in a Switch from Poleward Flux to Spindle Elongation. Proc Natl Acad Sci (2004) 101: 15938-43. doi:10.1073/pnas.0407044101

9. Lardennois A, Pásti G, Ferraro T, Llense F, Mahou P, Pontabry J, et al. An Actin-Based Viscoplastic Lock Ensures Progressive Body-axis Elongation. Nature (2019) 573:266-70. doi:10.1038/s41586-019-1509-4 structure formation, and mechanical response of the resulting network are discussed in detail.

The paper by Leong et al. presents coarse-grained molecular dynamics models of spike proteins in SARS-CoV-2 coronavirus and angiotensin-converting enzyme 2 (ACE2) receptor proteins to study the endocytosis of the virus under physiologically relevant spatial and temporal scales. The coarse-grained molecular simulations show that, during their interaction with the ACE2 receptors, the spike proteins adopt bent configurations because of their unique flexibility, which facilitates their attachment to the host cell membrane, compared with rigid spikes.

The paper by Mierke reviews the current understanding of the bidirectional mechanical interplay between cells and their microenvironment. Specifically, it points out how, on the one hand, the intracellular cytoskeletal architecture and, on the other hand, the matrix architecture contribute to cellular stiffness or contractility and thereby determines the emergence of a distinct migration mode of cells. In addition, it also discusses whether universal hallmarks of the migratory phenotype can be defined.

The paper by Strychalski presents a 3D dynamic computational model for describing cell blebbing where membrane locally detaches from the actin cortex, resulting in a pressure-driven flow of the cytosol and membrane expansion. In particular, bleb expansion dynamics under different initiation mechanisms are analyzed. Furthermore, the author also discusses the influence of treating the cytoplasm as a viscous fluid or as a poroelastic material on blebs' shape and growing speed.

\section{AUTHOR CONTRIBUTIONS}

\author{
This Editorial was prepared jointly by YLin and YLi.
}

10. Fang C, Wei X, Shao X, Lin Y. Force-mediated Cellular Anisotropy and Plasticity Dictate the Elongation Dynamics of Embryos. Sci Adv (2021) 7: eabg3264. doi:10.1126/sciadv.abg3264

11. Humphrey JD, Dufresne ER, Schwartz MA. Mechanotransduction and Extracellular Matrix Homeostasis. Nat Rev Mol Cel Biol (2014) 15:802-12. doi:10.1038/nrm3896

12. Engler AJ, Sen S, Sweeney HL, Discher DE. Matrix Elasticity Directs Stem Cell Lineage Specification. Cell (2006) 126:677-89. doi:10.1016/ j.cell.2006.06.044

13. Hui TH, Kwan KW, Chun Yip TT, Fong HW, Ngan KC, Yu M, et al. Regulating the Membrane Transport Activity and Death of Cells via Electroosmotic Manipulation. Biophysical J (2016) 110:2769-78. doi:10.1016/j.bpj.2016.05.011

14. Yellin F, Li Y, Sreenivasan VKA, Farrell B, Johny MB, Yue D, et al. Electromechanics and Volume Dynamics in Nonexcitable Tissue Cells. Biophysical J (2018) 114:2231-42. doi:10.1016/j.bpj.2018.03.033

15. Evans E, Heinrich V, Ludwig F, Rawicz W. Dynamic Tension Spectroscopy and Strength of Biomembranes. Biophysical J (2003) 85:2342-50. doi:10.1016/ s0006-3495(03)74658-x

16. Lin Y, Wei X, Qian J, Sze KY, Shenoy VB. A Combined Finite ElementLangevin Dynamics (FEM-LD) Approach for Analyzing the Mechanical Response of Bio-Polymer Networks. J Mech Phys Sol (2014) 62:2-18. doi:10.1016/j.jmps.2013.06.006

17. Onck PR, Koeman T, van Dillen T, van der Giessen E. Alternative Explanation of Stiffening in Cross-Linked Semiflexible Networks. Phys Rev Lett (2005) 95: 178102. doi:10.1103/physrevlett.95.178102 
18. Wei X, Zhu Q, Qian J, Lin Y, Shenoy VB. Response of Biopolymer Networks Governed by the Physical Properties of Cross-Linking Molecules. Soft Matter (2016) 12:2537-41. doi:10.1039/c5sm02820e

19. Cross SE, Jin Y-S, Rao J, Gimzewski JK. Nanomechanical Analysis of Cells from Cancer Patients. Nat Nanotech (2007) 2:780-3. doi:10.1038/nnano.2007.388

20. Suresh S, Spatz J, Mills JP, Micoulet A, Dao M, Lim CT, et al. Connections between Single-Cell Biomechanics and Human Disease States: Gastrointestinal Cancer and Malaria. Acta Biomater (2005) 1:15-30. doi:10.1016/j.actbio.2004.09.001

Conflict of Interest: The authors declare that the research was conducted in the absence of any commercial or financial relationships that could be construed as a potential conflict of interest.
Publisher's Note: All claims expressed in this article are solely those of the authors and do not necessarily represent those of their affiliated organizations, or those of the publisher, the editors and the reviewers. Any product that may be evaluated in this article, or claim that may be made by its manufacturer, is not guaranteed or endorsed by the publisher.

Copyright $\odot 2022$ Lin and Li. This is an open-access article distributed under the terms of the Creative Commons Attribution License (CC BY). The use, distribution or reproduction in other forums is permitted, provided the original author $(s)$ and the copyright owner(s) are credited and that the original publication in this journal is cited, in accordance with accepted academic practice. No use, distribution or reproduction is permitted which does not comply with these terms. 